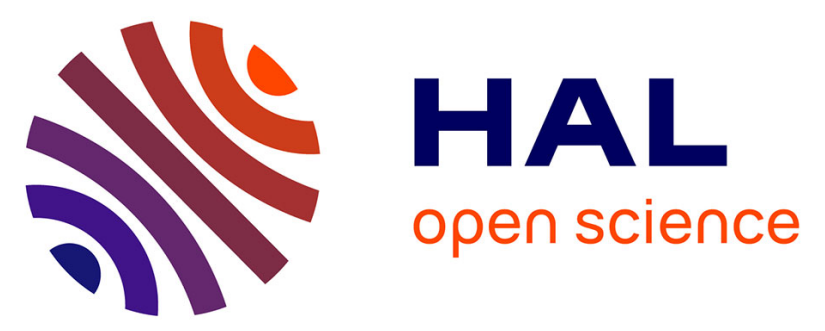

\title{
Pyrethroid insecticide exposure and cognitive developmental disabilities in children: The PELAGIE mother-child cohort
}

Jean-François Viel, Charline Warembourg, Gaïd Le Maner-Idrissi, Agnès Lacroix, Gwendolina Limon, Florence Rouget, Christine Monfort, Gaël

Durand, Sylvaine Cordier, Cécile Chevrier

\section{To cite this version:}

Jean-François Viel, Charline Warembourg, Gaïd Le Maner-Idrissi, Agnès Lacroix, Gwendolina Limon, et al.. Pyrethroid insecticide exposure and cognitive developmental disabilities in children: The PELAGIE mother-child cohort. Environment International, 2015, 82, pp.69-75. 10.1016/j.envint.2015.05.009 . hal-01162374

\section{HAL Id: hal-01162374}

\section{https://hal-univ-rennes1.archives-ouvertes.fr/hal-01162374}

Submitted on 15 Sep 2015

HAL is a multi-disciplinary open access archive for the deposit and dissemination of scientific research documents, whether they are published or not. The documents may come from teaching and research institutions in France or abroad, or from public or private research centers.
L'archive ouverte pluridisciplinaire HAL, est destinée au dépôt et à la diffusion de documents scientifiques de niveau recherche, publiés ou non, émanant des établissements d'enseignement et de recherche français ou étrangers, des laboratoires publics ou privés. 
Pyrethroid insecticide exposure and cognitive developmental disabilities in children: the PELAGIE mother-child cohort.

Jean-François Viel ${ }^{a, b}$, Charline Warembourg ${ }^{a}$, Gaïd Le Maner-Idrissi ${ }^{c}$, Agnès Lacroix ${ }^{c}$, Gwendolina Limon $^{d}$, Florence Rouget ${ }^{\mathrm{a}, \mathrm{e}}$, Christine Monfort ${ }^{\mathrm{a}}$, Gaël Durand ${ }^{\mathrm{d}}$, Sylvaine Cordier ${ }^{\mathrm{a}}{ }$ Cécile Chevrier $^{\mathrm{a}}$

${ }^{a}$ INSERM-IRSET n 1085, Epidemiological Research on Environment, Reproduction and Development, University of Rennes 1, Rennes, France

${ }^{b}$ Department of Epidemiology and Public Health, University Hospital, Rennes, France

${ }^{c}$ Research Centre for Psychology, Cognition and Communication, University of Rennes 2, Rennes, France

d LABOCEA Laboratory, Plouzané, France

e Department of Pediatrics, University Hospital, Rennes, France

* Corresponding author at:

INSERM-IRSET, Department of Epidemiology and Public Health, University Hospital

2, rue Henri Le Guilloux, 35033 Rennes, France.

E-mail: jean-francois.viel@univ-rennes1.fr

Telephone: +33 299289 827, Fax: +33 299289766

Keywords: Pyrethroid pesticides; neurodevelopment; cognitive abilities 


\section{ABSTRACT}

Pyrethroid insecticides are widely used in agriculture and in homes. Despite the neurotoxicity of these insecticides at high doses, few studies have examined whether lower-level exposures could adversely affect children's neurodevelopment.

The PELAGIE cohort included 3421 pregnant women from Brittany, France between 2002 and 2006. When their children reached their sixth birthday, 428 mothers from the cohort were randomly selected, successfully contacted and found eligible. A total of $287(67 \%)$ mothers agreed to participate with their children in the neuropsychological follow-up. Two cognitive domains were assessed by the Wechsler Intelligence Scale for Children: verbal comprehension and working memory. Five pyrethroid and two organophosphate insecticide metabolites were measured in maternal and child first-void urine samples collected between 6-19 gestational weeks and at 6 years of age, respectively. Linear regression models were used to estimate associations between cognitive scores and urinary pyrethroid metabolite concentrations, adjusting for organophosphate metabolite concentrations and potential confounders.

Maternal prenatal pyrethroid metabolite concentrations were not consistently associated with any children's cognitive scores. By contrast, childhood 3-PBA and cis-DBCA concentrations were both negatively associated with verbal comprehension scores $(P$-trend $=0.04$ and $P$-trend $<0.01$, respectively) and with working memory scores ( $P$-trend $=0.05$ and $P$-trend $<0.01$, respectively). No associations were observed for the three other childhood pyrethroid metabolite concentrations (4F-3-PBA, cis-DCCA, and trans-DCCA).

Low-level childhood exposures to deltamethrin (as cis-DBCA is its principal and selective metabolite), in particular, and to pyrethroid insecticides, in general (as reflected in levels of the 3-PBA metabolite) may negatively affect neurocognitive development by 6 years of age. Whatever their etiology, these cognitive deficits may be of importance educationally, because cognitive impairments in children interfere with learning and social development. Potential causes that can be prevented are of paramount public health importance. 


\section{Introduction}

The use of pyrethroid insecticides has increased substantially throughout the world over the past several decades, replacing organophosphate and carbamate insecticides, because of their chemical potency against many pests, their relatively low mammalian toxicity and their favorable environmental profiles (Schleier and Peterson, 2011). Pyrethroid insecticides are neurotoxic agents that disrupt the normal function of the peripheral nervous system by altering the permeability of excited nerve cells to sodium ions. Repetitive nerve impulses cause incoordination, convulsions, and paralysis in insects and other pests (Soderlund and Bloomquist, 1989).

It is commonly assumed that the major exposure pathway for pyrethroid insecticides in the general population is diet (Schettgen et al., 2002). However, their use in common household products, such as household and garden insecticides, pet sprays and shampoos, lice treatments, and mosquito repellents applied to clothing, may also lead to short episodes of exposure. Young children may receive greater exposure because they are closer to the floor and surfaces where insecticides may settle, have extensive hand-to-mouth contact, and are more likely to receive head lice treatments, as suggested by their higher urinary concentrations of pyrethroid insecticide metabolites compared with adolescents and adults (Barr et al., 2005). The major exposure routes for children are dietary ingestion of solid foods, followed by nondietary ingestion of dust, except in homes with frequent insecticide applications, in which case dermal absorption is more important than dietary ingestion (Morgan 2012).

In humans, pyrethroids are rapidly metabolized by ester hydrolysis and hydroxylation, primarily in the liver. The detoxified metabolites are eliminated by the kidneys for several days after exposure. Because pyrethroids are metabolized so rapidly, the concentrations of intact pyrethroids in serum or plasma are much lower than those of urinary metabolites, which are considered to reflect short-term exposures in low-exposure scenarios with the potential for misclassification (Bradman et al., 2005; Koureas et al., 2012). The major metabolites of pyrethroid insecticides detected in the urine are 3-PBA (a common metabolite of up to 20 synthetic pyrethroid insecticides), 4-F-3-PBA (a metabolite of the fluorine-substituted pyrethroid insecticide cyfluthrin), cis-DCCA and trans-DCCA (geometric isomeric metabolites of the chlorinated pyrethroid insecticides permethrin, cypermethrin, and cyfluthrin), and cis-DBCA (a selective metabolite of deltamethrin). 
The mode of action of pyrethroid insecticides raises concerns for human health. The potential effects of low-level chronic exposure to pyrethroid insecticides on neurobehavioral functioning are particularly relevant for fetuses and young children, who may be especially vulnerable to neurotoxic agents because of their immature nervous systems and their rapid rates of brain growth and development. A limited number of epidemiologic studies have examined the associations between maternal prenatal pyrethroid exposure and effects on neurodevelopment (Horton et al., 2011; Shelton et al., 2014). Recently, however, Oulhote and Bouchard (2013) reported a significant association between childhood concentration of cis-DCCA in urine and behavioral difficulties. Because pyrethroid and organophosphate (OP) insecticides are frequently encountered in the same environments, and because recent studies have provided compelling evidence for an association between prenatal OP insecticide exposure and neurodevelopmental dysfunction (Rauh et al., 2006; Eskenazi et al., 2007; Marks et al., 2010; Bouchard et al., 2011), potential confounding by OP exposure must be considered.

Using a longitudinal design, the aim of this study was to investigate associations between prenatal or childhood exposure to pyrethroid insecticides, as measured by urine pyrethroid metabolite concentrations, and cognitive abilities of 6-year-olds, after adjusting for OP insecticide metabolite levels during the corresponding measurement period.

\section{Methods}

\subsection{Study setting and design}

The PELAGIE cohort has been described previously (Petit et al., 2010; Chevrier et al., 2011). Briefly, 3,421 pregnant women from Brittany, France were included from January 2002 to February 2006. Women were enrolled before the $19^{\text {th }}$ week of gestation after completing a questionnaire at home concerning family, social and demographic characteristics, diet, and lifestyle. Midwives and pediatricians at the maternity units provided the study staff with medical information about the pregnancy, delivery, birth weight and neonatal health for 3,399 women and their newborns.

A random subcohort of 591 mothers was selected for pesticide determination in urine samples from the mothers who delivered live-born singleton infants, to obtain a final sample of size similar to those used in previous OP insecticide exposure studies (Rauh et al., 2006; Eskenazi et al., 2007). For the cognitive assessments at age 6 , exclusion criteria were length of pregnancy $<35$ weeks of amenorrhea, neonatal abnormalities (e.g., severe hypoglycemia, low Apgar score), neonatal hospitalization, and Down syndrome. Among the 571 eligible families, 446 were successfully 
contacted by phone and 18 were further excluded because of previous child neuropsychological or behavioral tests (to avoid bias due to the learning effect). A total of 287 (67\%) mothers agreed to participate with their child in the neuropsychological follow-up. Home visits were organized by two psychologists who were blinded to exposure status and supervised by four pediatric neuropsychologists in meetings held every two months. One of the psychologists completed child neurodevelopmental assessments, and the other psychologist was in charge of maternal interviews, maternal intelligence scoring, home environment assessments, child urine collections and dust sampling.

\subsection{Cognitive assessments at age 6}

The Wechsler Intelligence Scale for Children, $4^{\text {th }}$ edition (WISC-IV) was used to assess cognitive abilities (Wechsler, 2003). Scores were calculated for two domains: the Verbal Comprehension Index (WISC-VCI), which measures verbal concept formation and is a good predictor of school readiness, and the Working Memory Index (WISC-WMI), which assesses child ability to memorize new information, hold it in short-term memory, concentrate and manipulate information. Higher scores indicate better neurocognitive abilities.

\subsection{Maternal interviews and assessments at child age 6}

Mothers completed a self-administered questionnaire to provide information on sociodemographic characteristics, lifestyle factors, their child's health, and their child's environmental exposures. Mothers were also administered the Wechsler Adult Intelligence Scale $3^{\text {th }}$ revision (WAIS-III) (Wechsler, 1997). The Verbal Intelligence Quotient (VIQ) score was used to assess general knowledge, language, reasoning, and memory skills. To evaluate the quality and extent of stimulation available to the child in the home environment, the HOME (Home Observation for Measurement of the Environment) inventory was used as in many studies of neurotoxicity (Caldwell and Bradley, 1979). Higher HOME scores indicate a more supportive and stimulating home environment. We adapted the scale for today's French environments. Doubleblind scoring was performed on a random sample (39 homes) to monitor the accuracy of HOME inventory; 98\% concordance was observed among all dichotomous items.

\subsection{Laboratory methods}


To measure the highest possible concentrations, first-morning-void urine samples were collected, during early pregnancy (6-19 gestational weeks) for mothers and at the visit at 6 years of age for children (Barr et al., 2010). Upon arrival at the LABOCEA laboratory (Plouzané, France), urine samples were frozen at $-20^{\circ} \mathrm{C}$ until analysis. To minimize analytic heterogeneity, both the mother's and the child's pyrethroid concentrations were measured in the same batch of samples.

3-PBA and 4-F-3-PBA metabolites in 1-ml urine samples were extracted using an online solidphase extraction system Waters 2777C and Waters Oasis HLB Direct Connect cartridges with elution during the chromatography mobile phase. Separation was achieved by ultra-performance liquid chromatography (Acquity UPLC, Waters), using a Waters BEH C18 column (150 x $2.1 \mathrm{~mm}, 1.7 \mu \mathrm{m})$ and an elution gradient consisting of acetonitrile/formic acid $0.05 \%$ and water/formic acid $0.05 \%$. Detection relied on ultra-performance liquid chromatography and triple quadrupole mass spectrometry (UPLC/MS-MS) (Xevo TQ-S, Waters). Reference standards were provided by SigmaAldrich and Dr. Ehrenstorfer. Concentration range linearity was observed from $0.02 \mu \mathrm{g} / \mathrm{L}$ to $2 \mu \mathrm{g} / \mathrm{L}$ for 3-PBA and from 0.01 to $0.20 \mu \mathrm{g} / \mathrm{L}$ for 4-F-PBA. The coefficients of variation ranged from $19 \%$ to 20\%. Regarding cis-DCCA, trans-DCCA and cis-DBCA metabolites, a simultaneous extraction and derivatization was performed in 2-ml urine samples with Pentafluorobenzyl bromide (PFBBr) as alkylation reagent (Aldrich) and $1 \mathrm{ml}$ dichloromethane (Carlo Erba). DCCA-D9 (Dr Ehrenstorfer) was added as an internal standard. The organic phase was extracted and evaporated to $250 \mathrm{ml}$ using a Turbovap II system (Zymarck). The samples were analyzed by gas chromatography (7890 A, Agilent) and triple quadrupole spectrometry (7000 C, Agilent), using a Varian FactorFour VF-1 ms column $(15 \mathrm{~m} \times 0.25 \mathrm{~mm}, 0.1 \mu \mathrm{m})$. Concentration range linearity was observed from 0.011 to $1.08 \mu \mathrm{g} / \mathrm{L}$ for cis-DCCA, from 0.022 to $2.15 \mu \mathrm{g} / \mathrm{L}$ for trans-DCCA, and from 0.023 to $2.26 \mu \mathrm{g} / \mathrm{L}$ for cis-DBCA. The coefficients of variation ranged from $13 \%$ to $22 \%$.

Six nonspecific OP dialkylphosphate metabolites were measured in child urine using the same UPLC/MS-MS method. Three diethyl (DE) phosphate metabolite (diethylphosphate, diethylthiophosphate, diethyldithiophosphate) concentrations were summed, as well as three dimethyl (DM) phosphate metabolite (dimethylphosphate, dimethylthiophosphate, dimethyldithiophosphate) concentrations. Diethyl-thiophosphate-d10 and dimethylthiophosphated6 served as internal standards for non-specific organophosphate metabolites. Maternal OP metabolite levels were measured during pregnancy in $10 \mathrm{ml}$-urine samples with liquid chromatography and triple quadrupole mass spectrometry (LC/MS-MS) after solid-phase extraction, as previously described (Chevrier et al., 2011). The coefficients of variation ranged from $13 \%$ to $20 \%$. 
Eighty-two mothers were missing measures for all pyrethroid metabolites, and 55 mothers were missing measures for DM and DE phosphate metabolites, mostly because of samples entirely used for other urine assays. Three children were missing 3-PBA, 4-F-3PBA, DM and DE phosphate levels, and four were missing trans-DCCA, cis-DCCA and cis-DBCA measures.

Lead, which we considered to be a potential confounder (because of its neurotoxicity and the frequent lead-pesticide co-exposure in the home environment), was measured in wipe samples of floor dust collected in the living room using a standard protocol (ASTM E1792-03 - Standard Specification for Wipe Sampling Materials for Lead in Surface Dust). Samples were analyzed using an inductively-coupled plasma mass spectrometer (ICP/MS, Agilent Technology 7500ce) equipped with a quadrupole mass filter and an octopole reaction cell, with a limit of quantification of $2 \mu \mathrm{g} / \mathrm{m}^{2}$ for acid-leachable lead.

\subsection{Data analysis}

To preserve the size of the analytic sample, missing values for covariates were replaced by the modal value from participants with non-missing values. Imputation was required for 6 mothers (for a total of 6 values) and 14 children (for a total of 40 values). In addition, missing values for the neuropsychological scores were replaced by the values predicted from the subdomain scores when available (WISC-VCI=4, WISC-WMI=12, WAIS-III=2). As a result, the number of children with available neurocognitive scores was 287 for WISC-VCI and 283 for WISC-WMI.

Non parametric Spearman correlation coefficients were calculated between metabolite levels. Associations between cognitive scores (WISC-VCI or WISC-WMI as dependent variable) and maternal prenatal urinary pyrethroid metabolite concentrations were examined using multiple linear regression models. Prenatal metabolite levels were categorized into two groups for 3-BPA and 4-F-3-PBA (< limit of detection [LOD], $\geq$ LOD) and into three groups for cis-DCCA and cis-DBCA ( $\angle L O D$, and for those with a detectable level, subdivided below and above the median). Trans-DCCA levels were divided into tertiles. Maternal factors that were considered were the following: age (continuous), place of residence (rural, urban), parity $(0, \geq 1)$, pre-pregnancy body mass index $(\leq 25$, $>25 \mathrm{~kg} / \mathrm{m}^{2}$ ), education ( $\leq 12,>12$ years), WAIS-III VIQ (continuous), tobacco smoking at the beginning of pregnancy (no, yes), usual fish consumption before pregnancy $(<2, \geq 2$ times a week), length of pregnancy (continuous) and non-exclusive breastfeeding (none, $\leq 16,>16$ weeks). For the 6-year-old children, the following variables were considered: sex, birth weight (continuous), education (nursery school, primary school), number of siblings at age 6 (continuous), sleep duration 
$(<10.5,10.5-11,>11$ hours per day), duration of television watching $(<2.5,2.5-4.5,>4.5$ hours per week), duration of video gaming (0, 0-1.5, $\geq 1.5$ hours per week), extra-curricular sport activities (no, yes), and urinary cotinine concentration measured in the same urine samples as the pesticides $(<6, \geq 6 \mu \mathrm{g} / \mathrm{L})$. Finally, several environmental factors and exposures were also examined: HOME score when the child was 6 years of age (continuous), acid-leachable lead in the living room $(\leq 1,1$ $\left.3,>3 \mu \mathrm{g} / \mathrm{m}^{2}\right)$, number of smokers at home $(0,1, \geq 2)$, and cigarettes smoked at home $(0,0-10,>10$ per day). The psychologist who administered the psychological tests was also investigated as a potential source of measurement errors. We included a priori maternal education, WAIS-III VIQ score and HOME score in models because they are important determinants of children's cognitive development (Bouchard et al., 2011). DE and DM phosphate metabolites in maternal urine samples were forced into the models, to disentangle the potential neurotoxic effects of pyrethroid insecticides from that of organophosphate insecticides, as well as the childhood concentration of the pyrethroid metabolite under study, to account for its potential competing influence. In addition, we included urinary creatinine concentrations (for mothers and children) to account for urinary dilution. The remaining variables that predicted both the cognitive scores and the pyrethroid metabolite level with $p<0.2$ were retained as model covariates. Separate models were used to estimate associations with the five pyrethroid metabolites. Positive $\beta$ coefficients indicate better neurocognitive performance.

Tests for monotonic trend between metabolite levels and cognitive scores were performed using a reverse-scale Cox regression model recently proposed by Dinse et al. (2014) to handle nondetected values. In this method, the measured metabolite is treated as the modelled outcome, switching the roles of exposure and health effect. The method begins by reversing the concentration scale and then applying Cox regression analysis with non-detected values as rightcensored data. The same confounding variables as were used in the first approach were introduced into the Cox regression models.

The same confounder selection strategy was used to study associations between cognitive scores and childhood pyrethroid concentrations. Metabolite concentration categories differed slightly because the limits were based on childhood metabolite distributions. Childhood DE and DM concentrations were forced into the models. As with maternal prenatal concentrations, childhood pyrethroid metabolite concentrations were treated as categorical and as continuous (to carry out trend tests). 
$P$ Values $<0.05$ were considered statistically significant. Regression model diagnostics were performed and found to be acceptable. All statistical analyses were performed using R software ( $R$ Development Core Team 2014).

\subsection{Ethics Statement}

This study was approved by the French Consulting Committee for the Treatment of Information in Medical Research (no. 09.485) and by the French National Commission for the Confidentiality of Computerized Data (no. 909347). Written informed consent was obtained from each mother. Children provided verbal and witnessed assent.

\section{Results}

\subsection{Description of the population}

Demographic characteristics and lifestyle factors for the 287 mother-child pairs studied are reported in Table 1. At the beginning of their pregnancies, most mothers were $>27$ years of age, multiparous, and of healthy weight, had completed college and did not smoke. Children were predominantly attending a nursery school, slept at least 10.5 hours per day, had extra-curricular sport activities, and lived in a non-smoking environment.

The WISC-VCI scores ranged from 72 to $155(\mathrm{n}=287$, mean=106.76, 95\% confidence interval $[\mathrm{CI}]=$ 76.51-137.01), and the WISC-WMI scores ranged from 62 to 140 ( $n=283$, mean=106.77 [78.65134.89]).

\section{Table 1}

Socio-demographic and lifestyle factors of the study's mother-child pairs ( $n=287$, PELAGIE cohort, France).

\begin{tabular}{lcc}
\hline Characteristics & No. & $\%$ \\
\hline $\begin{array}{l}\text { Maternal factors } \\
\text { Age (years) }\end{array}$ & & \\
$\leq 27$ & 62 & 21.6 \\
$28-31$ & 131 & 45.6 \\
$\geq 32$ & 94 & 32.8 \\
Place of residence & & \\
rural & 158 & 55.1 \\
urban & 129 & 44.9 \\
Parity & & \\
0 & 122 & 42.5
\end{tabular}


Body mass index $\left(\mathrm{kg} / \mathrm{m}^{2}\right)$

$$
\leq 25
$$

Education (years)

$\leq 12$

91

31.7

$>12$

196

68.3

Tobacco smoking at the beginning of pregnancy

No

216

75.3

Yes

71

24.7

Child factors

Sex

Boys

139

48.4

Girls

149

51.6

Birth weight (grams)

$\begin{array}{lll}<3380 & 143 & 49.8 \\ \geq 3380 & 144 & 50.2\end{array}$

Education

Nursery school

214

74.6

Primary school

73

24.4

Sleep duration (hours per day)

$\begin{array}{lcc}<10.5 & 74 & 25.8 \\ 10.5-11 & 129 & 44.9 \\ >11 & 84 & 29.3\end{array}$

Extra-curricular sport

$\begin{array}{lll}\text { No } & 81 & 28.2\end{array}$

$\begin{array}{lll}\text { Yes } & 206 & 71.8\end{array}$

${ }^{a}$ For the sake of clarity, this variable is categorized in the table, but it was introduced into regression models as a continuous variable.

\subsection{Levels of urinary pyrethroid metabolites}

Table 2

Concentrations of pyrethroid insecticide urinary metabolites ( $\mu \mathrm{g} / \mathrm{L}$ ) (PELAGIE cohort, France).

\begin{tabular}{lcccccc}
\hline Exposure & No. & LOD & $\begin{array}{c}\text { Percent } \\
<\text { LOD }\end{array}$ & Median & $\begin{array}{c}\text { Median } \\
>\text { LOD }\end{array}$ & Maximum \\
\hline \multicolumn{2}{l}{ Prenatal (before the $19^{\text {th }}$} & week of gestation) & & & & \\
3-PBA & 205 & 0.008 & 69.8 & $<$ LOD & 0.051 & 0.518 \\
4-F-3-PBA & 205 & 0.003 & 91.2 & $<$ LOD & 0.079 & 0.722 \\
cis-DCCA & 205 & 0.067 & 35.1 & 0.090 & 0.138 & 3.660 \\
trans-DCCA & 205 & 0.010 & 2.0 & 0.140 & 0.141 & 2.390 \\
cis-DBCA & 205 & 0.067 & 31.7 & 0.105 & 0.150 & 2.450
\end{tabular}


Childhood (at 6 years of age)

\begin{tabular}{lllllll} 
3-PBA & 284 & 0.008 & 36.3 & 0.018 & 0.038 & 0.816 \\
4-F-3-PBA & 284 & 0.003 & 84.2 & $<$ LOD & 0.012 & 0.154 \\
cis-DCCA & 283 & 0.067 & 35.3 & 0.099 & 0.159 & 2.114 \\
trans-DCCA & 283 & 0.010 & 3.5 & 0.222 & 0.231 & 6.251 \\
cis-DBCA & 283 & 0.067 & 14.8 & 0.220 & 0.253 & 5.066 \\
\hline
\end{tabular}

LOD: limit of detection.

Table 2 presents the detection frequencies and distributions of the five pyrethroid metabolites measured in the maternal and child first-morning-void urine samples. Trans-DCCA, cis-DBCA and cis-DCCA metabolites were the most frequently detected species in both the mothers $199.9 \%$, $68.3 \%$ and $64.9 \%$, respectively) and the children (96.5\%, $85.2 \%$ and $64.7 \%$, respectively). Median concentrations followed broadly similar patterns.

Spearman correlation coefficients between the concentrations of different pyrethroid metabolites in maternal urine were high for the two DCCA isomers $\left(r_{s}=0.61\right)$ and moderate for DCCA isomers and cis-DBCA ( $r_{s}=0.35$ with cis-DCCA, and $r_{s}=0.39$ with trans-DCCA) and 3-PBA/transDCCA $\left(r_{s}=0.34\right)$. All other Spearman coefficients were $\leq 0.21$. A similar pattern was observed in the children at 6 years of age: DCCA isomers $\left(r_{s}=0.74\right)$, cis-DCCA/cis-DBCA $\left(r_{s}=0.35\right)$ and trans-DCCA/cisDBCA $\left(r_{s}=0.39\right)$. All other Spearman coefficients were $\leq 0.18$.

Four mother pyrethroid metabolite concentrations were uncorrelated with their child counterparts (3-PBA: $r_{s}=0.02 ; 4-F-3-P B A: r_{s}=0.04$; cis-DCCA: $r_{s}=-0.02 ;$ cis-DBCA: $r_{s}=0.01$ ), whereas trans-DCCA concentrations were moderately correlated $\left(r_{s}=0.24\right)$ with their child counterparts.

\subsection{Associations between maternal prenatal urinary levels of pyrethroid metabolites and child} neurocognitive scores

Table 3 presents the relations between maternal prenatal pyrethroid metabolite concentrations and neurocognitive scores, after adjusting for potential confounders of the associations, urinary creatinine levels, DM and DE prenatal concentrations, and the corresponding childhood pyrethroid metabolite concentrations. No consistent association was found, although some evidence suggested a potentially negative trend between trans-DCCA concentrations and WISC-WMI $(P$ trend=0.18). 
Table 3

Associations between maternal prenatal concentrations of pyrethroid urinary metabolites and Wechsler Intelligence Scale for Children (WISC-IV) scores (PELAGIE cohort, France). ${ }^{\text {a }}$

\begin{tabular}{|c|c|c|c|c|c|c|}
\hline \multirow[t]{2}{*}{ Pyrethroid metabolite } & \multicolumn{3}{|c|}{ Verbal comprehension index } & \multicolumn{3}{|c|}{ Working memory index } \\
\hline & No. & $\beta$ coefficient $[95 \% \mathrm{Cl}]$ & $P$-trend ${ }^{b}$ & No. & $\beta$ coefficient $[95 \% \mathrm{Cl}]$ & $P$-trend ${ }^{b}$ \\
\hline \multicolumn{7}{|l|}{ 3-PBA $(\mu \mathrm{g} / \mathrm{L})$} \\
\hline$<0.008^{\mathrm{c}}$ & 106 & - & 0.32 & 105 & - & 0.79 \\
\hline$\geq 0.008$ & 49 & $3.40[-1.83,8.62]$ & & 48 & $-0.11[-5.09,4.88]$ & \\
\hline \multicolumn{7}{|l|}{ 4-F-3-PBA ( $\mu \mathrm{g} / \mathrm{L})$} \\
\hline$<0.003^{\mathrm{c}}$ & 146 & - & 0.91 & 144 & - & 0.84 \\
\hline$\geq 0.003$ & 9 & $-0.15[-10.38,10.07]$ & & 9 & $-1.47[-11.10,8.15]$ & \\
\hline \multicolumn{7}{|l|}{$c i s-D C C A(\mu \mathrm{g} / \mathrm{L})$} \\
\hline$<0.067^{c}$ & 61 & - & 0.18 & 61 & - & 0.41 \\
\hline $0.067-0.137$ & 50 & $0.88[-5.10,6.86]^{d}$ & & 48 & $0.17[-5.51,5.85]^{d}$ & \\
\hline$\geq 0.138$ & 44 & $4.30[-2.26,10.85]^{d}$ & & 44 & $-3.56[-9.70,2.58]^{d}$ & \\
\hline \multicolumn{7}{|l|}{ trans-DCCA $(\mu \mathrm{g} / \mathrm{L})$} \\
\hline$<0.086$ & 53 & - & 0.24 & 53 & - & 0.18 \\
\hline $0.086-0.209$ & 48 & $-2.27[-8.38,3.83]^{\mathrm{e}}$ & & 48 & $-4.86[-10.48,0.76]$ & \\
\hline$\geq 0.210$ & 54 & $0.26[-5.68,6.20]^{\mathrm{e}}$ & & 52 & $-6.44[-11.92,-0.97]$ & \\
\hline \multicolumn{7}{|l|}{$c i s-\mathrm{DBCA}(\mu \mathrm{g} / \mathrm{L})$} \\
\hline$<0.067^{\mathrm{c}}$ & 51 & - & 0.81 & 51 & - & 0.64 \\
\hline $0.067-0.154$ & 52 & $0.12[-5.98,6.21]$ & & 51 & $-2.73[-8.58,3.13]$ & \\
\hline$\geq 0.155$ & 52 & $-0.41[-6.63,5.82]$ & & 51 & $-3.27[-9.31,2.77]$ & \\
\hline
\end{tabular}

${ }^{a}$ Maternal education, maternal Wechsler Adult Intelligence Scale score, Home Observation for Measurement of the Environment score, corresponding childhood pyrethroid metabolite concentration, urinary creatinine concentrations (mother and child), and detection of dimethyl (DM) and diethyl (DE) phosphates in maternal urine samples were forced into all models.

${ }^{\mathrm{b}}$ Provided by reverse-scale Cox regression models.

${ }^{c}$ Limit of detection.

${ }^{d}$ Adjusted for presence of acid-leachable lead in the living room.

${ }^{\mathrm{e}}$ Adjusted for maternal fish consumption. 
3.4. Associations between childhood urinary levels of pyrethroid metabolites and child neurocognitive scores

\section{Table 4}

Associations between childhood concentrations of pyrethroid urinary metabolites and Wechsler Intelligence Scale for Children (WISC-IV) scores (PELAGIE cohort, France). ${ }^{a}$

\begin{tabular}{|c|c|c|c|c|c|c|}
\hline \multirow{2}{*}{ Pyrethroid metabolite } & \multicolumn{3}{|c|}{ Verbal comprehension index } & \multicolumn{3}{|c|}{ Working memory index } \\
\hline & No. & $\beta$ coefficient $[95 \% \mathrm{Cl}]$ & $P$-trend ${ }^{b}$ & No. & $\beta$ coefficient $[95 \% \mathrm{Cl}]$ & $P$-trend ${ }^{b}$ \\
\hline \multicolumn{7}{|l|}{ 3-PBA ( $\mu \mathrm{g} / \mathrm{L})$} \\
\hline$<0.008^{c}$ & 103 & - & 0.04 & 103 & - & 0.05 \\
\hline $0.008-0.037$ & 90 & $-1.84[-5.89,2.22]^{d}$ & & 89 & $-1.56[-5.51,2.40]^{\mathrm{e}}$ & \\
\hline$\geq 0.038$ & 91 & $-5.18[-9.25,-1.11]^{d}$ & & 88 & $-3.90[-7.89,0.08]^{e}$ & \\
\hline \multicolumn{7}{|l|}{ 4-F-3-PBA ( $\mu \mathrm{g} / \mathrm{L})$} \\
\hline$<0.003^{\mathrm{c}}$ & 239 & - & 0.70 & 235 & - & 0.54 \\
\hline$\geq 0.003$ & 45 & $-0.50[-5.19,4.18]^{f}$ & & 45 & $1.66[-3.03,6.34]^{\mathrm{g}}$ & \\
\hline \multicolumn{7}{|l|}{ cis-DCCA ( $\mu \mathrm{g} / \mathrm{L})$} \\
\hline$<0.067^{c}$ & 100 & - & 0.82 & 100 & - & 0.28 \\
\hline $0.067-0.158$ & 91 & $0.26[-3.96,4.48]^{\mathrm{e}}$ & & 89 & $1.39[-2.66,5.44]^{\mathrm{h}}$ & \\
\hline$\geq 0.159$ & 92 & $1.08[-3.27,5.44]^{\mathrm{e}}$ & & 90 & $1.43[-2.80,5.66]^{\mathrm{h}}$ & \\
\hline \multicolumn{7}{|l|}{ trans-DCCA $(\mu \mathrm{g} / \mathrm{L})$} \\
\hline$<0.136$ & 94 & - & 0.76 & 92 & - & 0.91 \\
\hline $0.136-0.409$ & 94 & $-0.14[-4.41,4.13]^{i}$ & & 93 & $2.25[-1.77,6.26]^{\mathrm{j}}$ & \\
\hline$\geq 0.410$ & 95 & $-0.27[-4.74,4.19]^{i}$ & & 94 & $1.41[-2.86,5.67]^{\mathrm{j}}$ & \\
\hline \multicolumn{7}{|l|}{ cis-DBCA ( $\mu \mathrm{g} / \mathrm{L})$} \\
\hline$<0.134$ & 94 & - & $<0.01$ & 93 & - & $<0.01$ \\
\hline $0.134-0.345$ & 94 & $-2.05[-6.37,2.28]^{i}$ & & 92 & $-3.56[-7.84,0.71]^{d}$ & \\
\hline$\geq 0.346$ & 95 & $-6.75[-11.17,-2.32]^{i}$ & & 94 & $-3.94[-8.33,0.45]^{d}$ & \\
\hline
\end{tabular}




\footnotetext{
${ }^{a}$ Maternal education, maternal Wechsler Adult Intelligence Scale score, Home Observation for Measurement of the Environment score, corresponding childhood pyrethroid metabolite concentration, and detection of dimethyl (DM) and diethyl (DE) phosphates in maternal urine samples were forced into all models.

${ }^{\mathrm{b}}$ Provided by reverse-scale Cox regression models.

${ }^{c}$ Limit of detection.

${ }^{d}$ Adjusted for child duration of television watching and child extra-curricular sport activities.

${ }^{\text {e }}$ Adjusted for child extra-curricular sport activities.

${ }^{f}$ Adjusted for presence of acid-leachable lead in the living room.

${ }^{\mathrm{g}}$ Adjusted for maternal age and child sex, and presence of acid-leachable lead in the living room.

${ }^{h}$ Adjusted for maternal age, tobacco smoking at the beginning of pregnancy, number of siblings at age 6 , child extra-curricular sport activities and research psychologist.

'Adjusted for length of pregnancy, child duration of television watching and child extra-curricular sport activities. ${ }^{j}$ Adjusted for maternal age, number of siblings at age 6, child education, child duration of television watching, and child extra-curricular sport activities.

${ }^{k}$ Adjusted for length of pregnancy.
}

Table 4 reports results for childhood pyrethroid metabolite concentrations. For WISC-VCI scores, a negative association was observed with 3-PBA and cis-DBCA concentrations ( $P$-trend=0.04 and $P$ trend<0.01, respectively). Children exposed to the highest 3-PBA or cis-DBCA concentrations were more likely to experience lower WISC-VCI scores $(\beta=-5.18[-9.25,-1.11]$, and $\beta=-6.75[-11.17,-2.32]$, respectively) than children exposed to the lowest concentrations. Two covariates were associated with higher scores on the WISC-VCI in both multivariate 3-PBA and cis-DBCA models: maternal WAIS-III VIQ score $(\beta=0.38[0.19,0.56]$, and $\beta=0.35[0.17,0.54]$, respectively), and child extracurricular sport activities $(\beta=5.34[1.53,9.15]$, and $\beta=5.04[1.26,8.81]$, respectively) (Tables $A 2$ and A3).

For WISC-WMI scores, a negative association was found with 3-PBA and cis-DBCA concentrations $(P$-trend $=0.05$ and $P$-trend $<0.01$, respectively). Children with the highest urinary levels of 3-PBA scored lower on the WISC-WMI $(\beta=-3.90[-7.89,0.08])$ than did children with the lowest levels, whereas the pattern was less clear for cis-DBCA levels, as $\beta$ coefficients were similar for the second and third tertiles $(\beta=-3.56[-7.84,0.71]$, and $\beta=-3.94[-8.33,0.45]$, respectively). As for the WISC-VCl, two covariates were associated with higher scores on the WISC-WMI in both multivariate 3-PBA and cis-DBCA models: maternal WAIS-III VIQ score $(\beta=0.27[0.09,0.45]$, and $\beta=0.25[0.07,0.43]$, respectively) and child extra-curricular sport activities $(\beta=5.66[1.94,9.38]$, and $\beta=5.04[1.26,8.81]$, respectively) (Tables $\mathrm{A} 2$ and $\mathrm{A} 3$ ).

Finally, 4-F-3-PBA, cis-DCCA and trans-DCCA levels were neither associated with WISC-VCI scores nor with WISC-WMI scores (Table 4). 


\section{Discussion}

The results of this study show that childhood exposure to pyrethroid insecticides, as measured by urinary 3-PBA and cis-DBCA metabolite concentrations, was associated with poorer neurocognitive abilities in children at 6 years of age, after adjusting for various potential confounders and childhood DM and DE concentrations. No consistent associations were observed with maternal prenatal urinary pyrethroid metabolite concentrations.

The present study has many strengths, including its longitudinal design with pyrethroid exposure assessments both prenatally and during childhood. As outcomes, we used two domains from the WISC-IV known to be sensitive to low-dose insecticide exposure, providing targeted measures of possible neurotoxic effects on brain function (Bouchard et al., 2011; Rauh et al., 2011). Participants were representative of the PELAGIE cohort, although high-educated mothers were slightly more numerous (68\% vs. 62\%) (Petit et al., 2010). Moreover, their homogeneous socioeconomic profile (rather wealthy families, as in the whole cohort) may be seen as a strength, reducing the potential for uncontrolled confounding, as WISC-IV scores may be influenced by socioeconomic background (Wechsler, 2003). We used a sound and flexible statistical technique to handle biomarker values falling below LODs. The reverse-scale Cox regression model allows full use of the available data, is valid even with extreme LOD censoring, and does not assume any parametric distribution (Dinse et al., 2014). To minimize residual confounding we deliberately examined or adjusted for numerous risk factors, including known predictors of neurodevelopment factors. We also considered information about additional environmental neurotoxic exposures from substances such as organophosphate insecticides and lead. To provide robust results, no further backward selection was performed after entering some of these covariates into multivariate models. Finally, cognitively stimulating environments (as assessed by maternal WAIS-III VIQ score and child extra-curricular sport activities) that enhance intellectual development and skill acquisition in children (Weiss et al., 2006; Magnuson et al., 2009; Alloway and Alloway, 2010) were found significant in the multivariate models, in further support of our results.

Several limitations of this study should be noted. Assessing pyrethroid exposures in urine samples is challenging because of their fast clearance from the body, with complete excretion in the urine within a few days; thus, sample measurements reflect only acute or short-term exposures. Substantial within-subject variability has recently been observed in children (Attfield et al., 2014). Consequently, pyrethroid metabolites from spot urine samples may not represent a child's average exposure over time and may result in misclassification, reducing statistical power to detect associations. An additional concern about urinary biomarkers is that the metabolites in urine 
may reflect exposure to the metabolites themselves in the environment rather than to the parent compound (Morgan, 2012; Bradman and Whyatt, 2005). As both maternal and child pyrethroid metabolite levels were measured in one batch, we cannot rule out the possibility that a degradation of pyrethroid metabolites may have occurred during the 6 years in which the maternal urine samples were stored at $-20^{\circ} \mathrm{C}$. Moreover, as some child characteristics were not collected (e.g., history of head lice, history of pica), the possibility of residual confounding cannot be entirely dismissed. Finally, in the absence of firm mechanistic evidence linking brain anomalies to more refined neuropsychological testing, the observed functional deficits at 6 years of age should be interpreted with caution (Rauh et al., 2011).

In French PELAGIE children, 3-PBA concentrations were much lower (median value of $0.018 \mu \mathrm{g} / \mathrm{L}$ ) than in USA and German children (median values around $0.300 \mu \mathrm{g} / \mathrm{L}$ ) (Barr et al., 2010; Becker et al., 2006). This finding suggests a lower general usage of pyrethroid insecticides, as 3-PBA is considered a good overall nonspecific indicator of pyrethroid exposures. French children in the present study are likely predominantly exposed to pyrethroids that primarily metabolize to transDCCA (permethrin and cypermethrin, but not cyfluthrin because of the low frequency of detection of 4-F-3-PBA) and cis-DBCA (deltamethrin). Comparison of levels of urinary pyrethroid metabolites in children from the PELAGIE cohort and from the Canadian Health Measures Survey confirms these contrasting results (Oulhote and bouchard, 2013). In France, the median concentration is lower for 3-PBA $(0.018 \mu \mathrm{g} / \mathrm{L}$ vs. $0.200 \mu \mathrm{g} / \mathrm{L})$ but higher for cis-DBCA $(0.220 \mu \mathrm{g} / \mathrm{L}$ vs. $<0.006 \mu \mathrm{g} / \mathrm{L})$ compared to Canadian children.

Information regarding the potential developmental neurotoxicity of pyrethroid insecticides is limited, as acknowledged in the review by Shafer et al. (2005). Moreover, direct comparison with existing studies is complicated by differences in the exposure scenario, measurement methods and neurodevelopmental assessment methods. Shelton et al. showed that children of mothers residing near pyrethroid insecticide applications just prior to conception or during their third trimester were at greater risk for both autism spectrum disorders and developmental delays (Shelton et al., 2014). Horton et al. found no association between prenatal exposure to permethrin in personal air and/or plasma and neurodevelopment at 36 months (using performance scores for the Bayley Mental Developmental Index or the Psychomotor Developmental Index) (Horton et al., 2011). Regarding childhood exposures, our results are broadly in line with those of Oulhote and Bouchard (2013) who observed a significant association between childhood concentration of cis-DCCA in the urine (but not 3-PBA concentrations, whereas cis-DBCA was not considered) and high scores for total behavioral difficulties on the Strengths and Difficulties Questionnaire. On the other hand, Quirós- 
Alcalá et al. (2014) reported that childhood pyrethroid exposure was not associated with parental reports of learning disabilities or attention-deficit/hyperactivity disorder.

In conclusion, low-level childhood exposures to deltamethrin (as cis-DBCA is its principal and selective metabolite), in particular, and to pyrethroid insecticides, in general (as reflected in levels of the 3-PBA metabolite) may negatively affect neurocognitive development by 6 years of age. Whatever their etiology, these cognitive deficits may be of importance educationally, because cognitive impairments in children interfere with learning and social development. Potential causes that can be prevented are of paramount public health importance.

\section{Conflict of interest}

All authors declare no actual or potential conflict of financial or other interests.

\section{Acknowledgment}

We are grateful to the gynecologists, obstetricians, ultrasonographers, midwives, pediatricians, and families who participated in the study. We thank Véronique Villalon and Catherine Nouyrigat for their help and Mathilde Mordelet and Olivia Martin for conducting neuropsychological testing.

This study was supported by the French National Research Agency (ANR-2010-PRSP-007), the French Pfizer Foundation and the French Research Institute for Public Health (AMC11004NSA-DGS). The funders had no role in the design or conduct of this study, the analysis or interpretation of the data, or the preparation of this manuscript. 


\section{References}

Alloway TP, Alloway RG. Investigating the predictive roles of working memory and IQ in academic attainment. J Exp Child Psychol 2010;106:20-9.

Attfield KR, Hughes MD, Spengler JD, Lu C. Within- and between-child variation in repeated urinary pesticide metabolite measurements over a 1-year period. Environ Health Perspect 2014;122:201-6.

Barr DB, Wilder LC, Caudill SP, Gonzalez AJ, Needham LL, Pirkle JL. Urinary creatinine concentrations in the U.S. population: Implications for urinary biologic monitoring measurements. Environ Health Perspect 2005;113:192-200.

Barr DB, Olsson AO, Wong LY, Udunka S, Baker SE, Whitehead RD, et al. Urinary concentrations of metabolites of pyrethroid insecticides in the general U.S. population: National Health and Nutrition Examination Survey 1999-2002. Environ Health Perspect 2010;118:742-8.

Becker K, Seiwert M, Angerer J, Kolossa-Gehring M, Hoppe HW, Ball M, et al. GerES IV pilot study: assessment of the exposure of German children to organophosphorus and pyrethroid pesticides. Int J Hyg Environ Health 2006;209:221-33.

Bouchard MF, Chevrier J, Harley KG, Kogut K, Vedar M, Calderon N, et al. Prenatal exposure to organophosphate pesticides and IQ in 7-year old children. Environ Health Perspect 2011;119:1189-95.

Bradman A, Whyatt RM. Characterizing exposures to nonpersistent pesticides during pregnancy and early childhood in the National Children's Study: a review of monitoring and measurement methodologies. Environ Health Perspect 2005;113:1092-9.

Bradman A, Eskenazi B, Barr DB, Bravo R, Castorina R, Chevrier J, et al. Organophosphate urinary metabolite levels during pregnancy and after delivery in women living in an agricultural community. Environ Health Perspect 2005;113:1802-7.

Caldwell BM, Bradley RH. Home observation for measurement of the environment. Little Rock, AR (USA): University of Arkansas Press; 1979.

Chevrier C, Limon G, Monfort C, Rouget F, Garlantézec R, Petit C, et al. Urinary biomarkers of prenatal atrazine exposure and adverse birth outcomes in the PELAGIE birth cohort. Environ Health Perspect 2011;119:1034-41. 
Dinse GE, Jusko TA, Ho LA, Annam K, Graubard BI, Hertz-Picciotto I, et al. Accommodating measurements below a limit of detection: a novel application of Cox regression. Am J Epidemiol 2014;179:1018-24.

Eskenazi B, Marks AR, Bradman A, Harley K, Barr DB, Johnson C, et al. Organophosphate pesticide exposure and neurodevelopment in young Mexican-American children. Environ Health Perspect 2007;115:792-8.

Horton MK, Rundle A, Camann DE, Boyd Barr D, Rauh VA, Whyatt RM. Impact of prenatal exposure to piperonyl butoxide and permethrin on 36-month neurodevelopment. Pediatrics 2011;127:e699-e706.

Koureas M, Tsakalof A, Tsatsakis A, Hadjichristodoulou C. Systematic review of biomonitoring studies to determine the association between exposure to organophosphorus and pyrethroid insecticides and human health outcomes. Toxicol Lett 2012;210:155-68.

Le Bot B, Arcelin C, Briand E, Glorennec P. Sequential digestion for measuring leachable and total lead in the same sample of dust or paint chips by ICP-MS. J Environ Sci Health A Tox Hazard Subst Environ Eng 2011;46:63-9.

Magnuson K A, Sexton H R, Davis-Kean P E, Huston AC. Increases in maternal education and young children's language skills. Merrill-Palmer Quart 2009;55:319-50.

Marks AR, Harley K, Bradman A, Kogut K, Barr DB, Johnson C, et al. Organophosphate pesticide exposure and attention in young Mexican-American children: the CHAMACOS study. Environ Health Perspect 2010;118:1768-74.

Morgan MK. Children's exposures to pyrethroid insecticides at home: a review of data collected in published exposure measurement studies conducted in the United States. Int J Environ Res Publ Health 2012;9:2964-85.

Oulhote Y, Bouchard MF. Urinary metabolites of organophosphate and pyrethroid pesticides and behavioral problems in Canadian children. Environ Health Perspect 2013;121:1378-84.

Petit C, Chevrier C, Durand G, Monfort C, Rouget F, Garlantezec R, et al. Impact on fetal growth of prenatal exposure to pesticides due to agricultural activities: a prospective cohort study in Brittany, France. Environ Health 2010;15;9:71.

Quirós-Alcalá L, Mehta S, Eskenazi B. Pyrethroid Pesticide Exposure and Parental Report of Learning Disability and Attention Deficit/Hyperactivity Disorder in U.S. Children: NHANES 1999-2002. Environ Health Perspect 2014;122:1336-42. 
Rauh VA, Garfinkel R, Perera FP, Andrews HF, Hoepner L, Barr DB, et al. Impact of prenatal chlorpyrifos exposure on neurodevelopment in the first 3 years of life among inner-city children. Pediatrics 2006;118:e1845-e1859.

Rauh V, Arunajadai S, Horton M, Perera F, Hoepner L, Barr DB, et al. Seven-year neurodevelopmental scores and prenatal exposure to chlorpyrifos, a common agricultural pesticide. Environ Health Perspect 2011;119:1196-201.

Shafer TJ, Meyer DA, Crofton KM. Developmental neurotoxicity of pyrethroid insecticides: critical review and future research needs. Environ Health Perspect 2005;113:123-36.

Shelton JF, Geraghty EM, Tancredi DJ, Delwiche LD, Schmidt RJ, Ritz B, et al. Neurodevelopmental disorders and prenatal residential proximity to agricultural pesticides: The CHARGE Study. Environ Health Perspect 2014;122:1103-9.

Schleier III JJ, Peterson RKD. Pyrethrins and pyrethroid insecticides. In: Óscar López O, FernándezBolaños J, editors. Green trends in insect control. Cambridge (UK): RSC Publishing; 2011. p. 94131.

Schettgen T, Heudorf U, Drexler H, Angerer J. Pyrethroid exposure of the general population - is this due to diet? Toxicol Lett 2002;134:141-5.

Soderlund DM, Bloomquist JR. Neurotoxic actions of pyrethroid insecticides. Annu Rev Entomol 1989;34:77-96.

Wechsler D. Wechsler Adult Intelligence Scale-III: Administration and scoring manual. 3rd ed. San Antonio, TX (USA): The Psychological Corporation; 1997.

Wechsler D. Wechsler Intelligence Scale for Children: Administration and scoring manual. 4th ed. San Antonio, TX (USA): Harcourt Assessment; 2003.

Weiss LG, Harris JG, Prifitera A, Courville T, Rolfhus E, Saklofske DH et al. WISC-IV interpretation in societal context. In: Weiss LG, Saklofske DH, Prifitera A, Holdnack JA, editors. WISC-IV: Advanced clinical interpretation. Burlington, MA (USA): Academic Press/Elsevier; 2006. p. 1-58. 